\title{
Reviewer Acknowledgements for Global Journal of Health Science, Vol. 8, No. 10
}

Global Journal of Health Science wishes to acknowledge the following individuals for their assistance with peer review of manuscripts for this issue. Their help and contributions in maintaining the quality of the journal are greatly appreciated.

Global Journal of Health Science is recruiting reviewers for the journal. If you are interested in becoming a reviewer, we welcome you to join us. Please find the application form and details at http://recruitment.ccsenet.org and e-mail the completed application form to gjhs@ccsenet.org.

\section{Reviewers for Volume 8, Number 10}

A. Kadir Mutlu

A. C. O. Maia

A. Mazaherinezhad

Akansha Tyagi

Ana I. Cobo Cuenca

Ann Noseworthy

Anna Ali

Béfa Noto Kadou Kaza

Bogdali M. Anna

Celia Moffat Joel Matyanga

Chiara Rapallini

Chung-Yu Chen

Cinara Sacomori

Claire de Labrusse

Daleep Kumar Arora

Darampal Dambhare

Dave A. Bergeron

Domitila Huber

Eiichi Ishikawa

Emille Joana Medeiros Capistrano

Esteban Ortiz-Prado

Faik Ardahan

Farhana Abedin

Feifei Chen

Funda Tosun Güleroğlu

Gabriele Messina

George Giannakoulas

Glenn L Sia Su

Habib Besbes

Haleh Dadgostar

Harsimran Kaur

Hetron Mweemba Munang'andu

Izabel Cristina Rodrigues da Silva
Jason Tsai

Jichuan Wang

João Gregório

Johannes J. Sidelmann

Kristian Hoelscher

L.C. Mourão

Li Guangwu

Linda $\mathrm{Ng}$

Liye Suo

Ljubica Djukanović

Lobna A. Aly

Magdalena Pertynska-Marczewska

Maja Racic

Manal Ibrahim Mahmoud

Manuela Mauceri

Mao-Qiang Man

Mark A.Tully

Marla E. Lujan

Marta Berra

Mojgan Mirghafourvand

Monia Ben Khaled

Monia Ouederni

Myriam Razgallah Khrouf

Naesinee Chaiear

Osmar Nascimento Silva

Panayiotis C. Petrou

Pavol Mikula

Peter Pryzbylkowski

Plesa Roxana

Polly Yeung

Pradnya V. Kakodkar

Rahime Renda

Raildo Coqueiro 
Rebecca Kurnik Seshasai

Robert Eley

Rod MacLeod

Rui Li

Ryan S. Falck

Serge Brand

Sheeja S. Varghese

Shilpee Singh

Snehal Padhye

Sunmoo Yoon

Talli Y. Rosenbaum

Thomas Wenzel

Tom Møller

Toshiyuki Yasui

Wafaa F. Sleem

Weam Banjar

Yasam Kemal Akpak

Yong-Shian Goh

Yuan-Han Huang

Yuanyuan $\mathrm{Gu}$ 